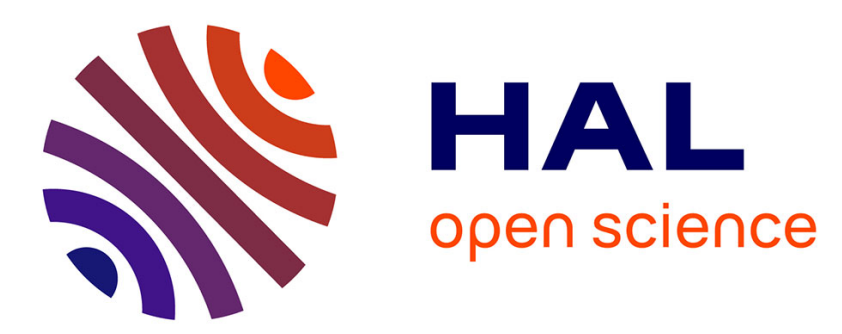

\title{
Évolution des paramètres de la marche sous traitement par biomédicaments au cours des rhumatismes inflammatoires chroniques
}

Germain Jelin

\section{- To cite this version:}

Germain Jelin. Évolution des paramètres de la marche sous traitement par biomédicaments au cours des rhumatismes inflammatoires chroniques. Rhumatologie et système ostéo-articulaire. 2016. dumas01388123

\section{HAL Id: dumas-01388123 https://dumas.ccsd.cnrs.fr/dumas-01388123}

Submitted on 26 Oct 2016

HAL is a multi-disciplinary open access archive for the deposit and dissemination of scientific research documents, whether they are published or not. The documents may come from teaching and research institutions in France or abroad, or from public or private research centers.
L'archive ouverte pluridisciplinaire HAL, est destinée au dépôt et à la diffusion de documents scientifiques de niveau recherche, publiés ou non, émanant des établissements d'enseignement et de recherche français ou étrangers, des laboratoires publics ou privés. 


\section{ÉVOLUTION DES PARAMÈTRES DE LA MARCHE SOUS TRAITEMENT PAR BIOMÉDICAMENTS AU COURS DES RHUMATISMES INFLAMMATOIRES CHRONIQUES}

Thèse de doctorat de médecine (Diplôme d'état) diplôme d'étude spécialisée en rhumatologie.

Présentée et soutenue publiquement le 13 JUIN 2016 par

\section{Germain JELIN}

Jury :

Président du Jury:

Monsieur le Professeur Patrice FARDELLONE (Rhumatologie, CHU Amiens)

Directeur de Thèse :

Monsieur le Professeur Vincent GOËB (Rhumatologie, CHU Amiens)

Membres du Jury

Monsieur le Professeur Patrice MERTL (Orthopédie, CHU Amiens)

Monsieur le Professeur Pierre-Louis DOUTRELLOT (Médecine Physique et de Réadaptation, CHU Amiens)

Monsieur le Docteur Bernard AUVINET (Rhumatologie, Polyclinique du Maine, Laval) 



\section{Dédicaces et Remerciements}

\section{Monsieur le Professeur Patrice FARDELLONE}

Professeur des Universités-Praticien Hospitalier (Rhumatologie)

Chef du service de Rhumatologie, Pôle « Autonomie ».

Cher Président, Cher Maître,

Je vous remercie de présider le jury de cette thèse. Depuis le début de mon internat, vous n'avez cessé de m'encourager à me perfectionner tout en me transmettant la passion qu'est la vôtre pour la rhumatologie grâce à votre savoir et votre expérience. Votre éternelle bonne humeur et votre relation avec le patient ont toujours été pour moi un modèle dans ma pratique de la médecine au quotidien.

\section{Monsieur le Professeur Patrice MERTL}

Professeur des Universités-Praticien Hospitalier

Chef du Service d'Orthopédie et traumatologie.

Membre associé de l'Académie de Chirurgie.

Responsable du Pôle «Autonomie ».

Cher Juge, Cher Maître,

Je vous remercie d'accepter de juger mon travail et de votre disponibilité. Le fait que nos deux spécialités soient toujours en étroite collaboration dans la rigueur et la probité est une chose indispensable à mes yeux que je m'efforcerai de maintenir tout au long de ma carrière.

\section{Monsieur le Professeur Pierre-Louis DOURTELLOT}

Professeur des Universités-Praticien Hospitalier (Médecine Physique et de Réadaptation), Responsable du Centre d'activité MPR Orthopédie

Cher Juge, Cher Maître,

Je vous remercie de l'honneur que vous me faites de juger mon travail. L'importance de la Médecine physique et de Réadaptation dans ma thèse montre à quel point nos deux spécialités sont liées, et je m'efforcerai au cours de ma carrière de pérenniser cette collaboration. 


\section{Monsieur le Professeur Vincent GOËB}

Professeur des Universités-Praticien Hospitalier (Rhumatologie)

Cher Directeur, cher Maître,

Je vous remercie de m'avoir attribué ce sujet si intéressant, si particulier et d'avoir accepté de diriger ma thèse.

Je vous remercie pour ce que vous m'avez enseigné tout au long de ces années, pour le temps que vous avez consacré à me former, pour toutes les valeurs inculquées et qui resterons gravées à jamais dans ma pratique de tous les jours. Vous avez su être présent dans les moments difficiles avec humanité, compréhension et vous avez fait preuve d'un soutien rare. Vous m'avez toujours guidé dans mon cursus de la meilleure manière qui soit.

\section{Monsieur le Docteur Bernard AUVINET}

Médecin Rhumatologue

Cher Juge,

Je vous remercie de m'avoir transmis votre passion pour l'étude des troubles de la marche, du temps passé à mettre en valeur ma thèse et de votre disponibilité pour venir participer à mon Jury malgré la distance. Je m'efforcerai, tout au long de ma carrière, de prolonger votre enseignement et votre savoir-faire concernant ce domaine. 


\section{Ce travail est aussi dédié,}

\section{A ma fiancée, Nathalie,}

Ton amour et ton soutien indéfectible durant ces années m'ont permis de relever chaque défi, et pour cela je t'en serai éternellement reconnaissant.

\section{A ma mère,}

Ton éducation, tes valeurs, ainsi que ton aide m'ont permis de toujours accomplir ce que j'avais entrepris, tu m'as transmis ton courage et j'espère en être digne tout au long de ma carrière.

\section{A mon père,}

Qui a toujours été présent à tous les moments et qui me rends fier chaque jour.

A mon frère, ma sœur, mes grands-parents,

Qui ont toujours été de véritables alliés dans ma vie quotidienne.

\section{A ma future belle famille,}

Puisse votre gentillesse continuer à illuminer la vie des gens.

\section{Aux disparus.}

\section{A tous les médecins qui ont contribué à ma formation,}

Dr Grados (merci pour tous ces conseils et pour toute votre pédagogie), Dr Lasselin-Boyard (merci pour ta bonne humeur, ta patience et ton aide à la rédaction d'articles médicaux), Dr Rehailia-Dehamchia (merci pour ta gentillesse et ta disponibilité), Dr Bellony, Dr DesaillyHenry, Dr Millot, Dr Boutry, Dr Fechtembaum, Dr Sejourne.

\section{Aux internes de rhumatologie du service, tout particulièrement,}

Mme Salomon, Mr Candelier, Mr Batteux, Mr Rabbin.

A tout le personnel médical, para-médical, aux agents des services hospitaliers, aux secrétaires du service et à Mme Barbier (responsable de la scolarité) pour leur travail et leur abnégation. 



\section{Sommaire}

I) Introduction

a. Le cycle de la marche (p.9)

b. Mesure des paramètres de la marche chez les patients atteints de rhumatismes inflammatoires chroniques (RIC) (p.10)

c. Troubles de la marche chez les patients atteints de spondylarthrite ankylosante (SA) (p.11)

d. Troubles de la marche chez les patients atteints de polyarthrite rhumatoïde (PR) (p.12)

e. L'accéléromètrie (p.14)

f. Etude accéléromètrique des variations des troubles de la marche chez les patients atteints de PR et de SA après la mise en place d'un biomédicament (p.16)

II) Patients et méthodes
a. Sélection (p.17)
b. Intervention (p.17)
c. Evaluation (p.18)
d. Ethique (p.18)

III) Résultats

a. Données observées à l'inclusion des patients (p.19)

b. Données observées après traitement (p.19)

IV) Discussion

a. Concernant les SA (p.20)

b. Concernant les PR (p.22)

V) Conclusion (p.23)

VI) Annexes (p.24)

VII) Bibliographie (p.34) 


\section{I) Introduction}

a. Le cycle de la marche

Les premières analyses de la marche remontent au XIXe siècle, développées par Marey et Muybridge [1,2]. Les quatre principales articulations intervenant dans son développement sont l'articulation coxo-fémorale, le genou, l'articulation talo-crurale et les articulations du tarse. Une stride (foulée en français) correspond à un pas, lui-même constitué du demi pas droit et du demi pas gauche. Un cycle de marche est composé de deux demi-pas. Il est divisé en deux phases [Figure 1] :

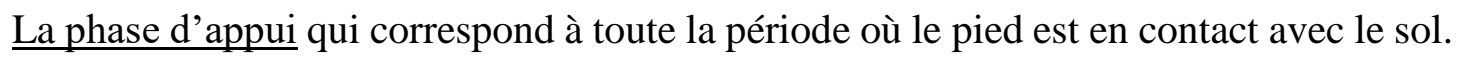
Son début correspond au contact initial (contact du pied avec le sol) et sa fin au décollement des orteils (perte de contact du pied avec le sol). Elle s'étend de $0 \%$ à $60 \%$ du cycle de marche et elle est également divisée en trois phases :

Le double appui de réception (10\% du cycle) : les deux pieds sont en contact avec le sol, le pied qui atterrit se pose entièrement avec une rotation externe d'environ $10^{\circ}$ par rapport à la direction du déplacement, la cheville effectue d'abord une flexion neutre, puis une flexion plantaire de 5 à $10^{\circ}$, le genou et la hanche fléchissent pour amortir le choc, respectivement de 8-12 ${ }^{\circ}$ et de 3$5^{\circ}$ pendant que le pied control-latéral quitte progressivement le sol.

La position plantigrade (40\% du cycle) : il s'agit de la phase d'appui monopodale, le pied est en rotation externe de $5-10^{\circ}$, $1^{\prime}$ articulation talo-crurale effectue alors une flexion dorsale de $10^{\circ}$, le genou et la hanche sont parvenus en extension.

Le double appui de propulsion (10\% du cycle) : les deux pieds sont en contact avec le sol, la tête du premier métatarsien pivote ; il en découle un début de flexion plantaire et de rotation externe du pied qui sont maximales en début de phase oscillante. Le genou et la hanche sont en extension $\left(5-10^{\circ}\right)$ et $\left(-5^{\circ} \grave{a}-10^{\circ}\right)$.

La phase oscillante où le pied n'est plus en contact avec le sol ce qui permet l'avancée du membre inférieur. Son début commence par le décollement des orteils et se termine lors du contact au sol suivant du même pied. Elle s'étend de 60 à $100 \%$ du cycle de marche, soit une durée de $40 \%$ du cycle de marche repartie également en 3 phases.

L'oscillation initiale : le pied est décollé du sol et en rotation externe maximale (environ $20^{\circ}$ ), la cheville est en flexion plantaire maximale (10-20 $)$, le genou est en flexion maximale (60$70^{\circ}$ ), la hanche fléchie. Le membre oscillant va croiser le membre porteur. 
L'oscillation intermédiaire : le pied tourne vers l'intérieur, la cheville réduit sa flexion plantaire, le genou débute une extension, la hanche tend vers sa flexion maximale. Le membre oscillant croise le membre porteur.

L'oscillation terminale : le pied est en légère rotation externe (environ $10^{\circ}$ ), la cheville est en flexion neutre, la hanche a atteint sa flexion maximale $\left(30^{\circ}\right)$. Le membre oscillant se pose sur le sol.

b. Mesure des paramètres de la marche chez les patients atteints de rhumatismes inflammatoires chroniques (RIC)

Enregistrement cinétique sur parcours : Différents appareils ont été utilisés dans la littérature afin de quantifier la marche chez les patients atteints de polyarthrite rhumatoïde (PR). Frensen et al [3] utilisaient une passerelle de 8 mètres, avec un appareillage métallique fixé sur les chaussures, relié à un câble électrique qui lui-même, grâce à un système de poulies, envoyait les données enregistrées à un micro-ordinateur. Le principal défaut de ce système était la taille insuffisante de la passerelle qui ne permettait pas réellement d'enregistrer une marche stabilisée, ni de s'affranchir de l'accélération et de la décélération en fin de parcours. Seules la vitesse, la cadence et la longueur du pas étaient enregistrées par cet appareil. Eppeland et al [4] employaient le «GAITRite walkway system » pour mesurer les mêmes paramètres chez les patients atteints de PR : un tapis de 7 mètres muni de capteurs placés sur les 4,3 mètres au centre de ce tapis. Il existait toujours une distance trop courte pour enregistrer une marche stabilisée, mais ce système était moins encombrant pour le patient que celui cité précédemment (aucun matériel fixé au patient) permettant une mesure, non biaisée par le matériel des mêmes paramètres. A noter que dans cette étude, les sujets étaient comparés après plusieurs parcours effectués à différentes vitesses.

Les systèmes de captures de mouvements pour les enregistrements cinématiques : que ce soit dans les spondylarthrites ankylosantes (SA) [5,6] ou dans la PR [7-9], la mesure du mouvement de marche par des systèmes de captures de mouvements est très répandue. Bien que coûteuse, cette technique permet non seulement de mesurer les paramètres cinétiques de la marche mais elle permet également de s'intéresser aux paramètres cinématiques (études dynamiques des amplitudes articulaires, moments articulaires, etc...). Cela a permis de développer de nouveaux scores («Gait deviation index») et de mieux approcher les troubles de la marche en trois dimensions chez les patients atteints de rhumatismes inflammatoires à chaque moment du cycle de la marche. 
Les plateformes de forces : Couplé aux systèmes de captures de mouvements $[5,9,10]$ ou seul [11-13], ce système permet de calculer des données statiques posturales telles que le centre de gravité et le centre de pression (correspondant à la force de réaction du sol au poids du sujet). Il est utilisé en double tache : les yeux ouverts et fermés.

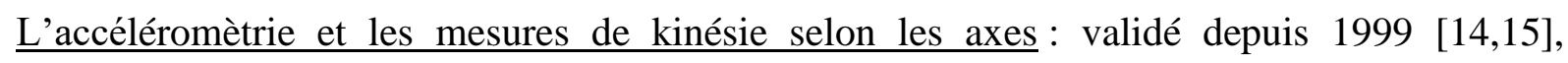
l'accéléromètre permet, en plus des données cinétiques de la marche, de pouvoir calculer la kinésie du mouvement dans les 3 axes (antéro-postérieur, cranio-caudal et médio-latéral). Cette technique a été utilisée récemment pour comparer les performances de locomotions entre les patients atteints de PR et de SA et un groupe contrôle. On note également que l'accéléromètre miniature a été utilisé [16] afin de déterminer l'origine rhumatoïde ou spondylarthritique d'une arthrite du genou, permettant d'isoler les puissances d'accélération comme facteur potentiellement discriminant.

c. Troubles de la marche chez les patients atteints de spondylarthrite ankylosante (SA)

\section{D’un point de vue statique :}

Les SA rachidiennes avec ou sans atteinte périphérique sont des rhumatismes inflammatoires dont la physiopathogénie exacte reste encore non élucidée. Elle est fortement associée à la présence de l'antigène $H L A-\mathrm{B} 27$ et à d'autres gènes encore en cours d'identification. Les atteintes chez des patients atteints de SA axiale et périphérique sont bien connues, avec une atteinte inflammatoire des articulations sacro-iliaques et vertébrales responsables respectivement de fusions des berges articulaires (sacro-iliite ou ankylose) et de syndesmophytes pouvant conduire à la typique «colonne bambou». D'un point de vue rachidien il y a une disparition de la lordose lombaire et une aggravation de la cyphose thoracique ; on note également l'apparition possible d'une cyphose cervicale [17] responsable d'une protrusion en avant de la tête et d'une diminution des amplitudes articulaires de la région du cou. Les patients sont alors, dans les formes les plus avancées, incapables de regarder l'horizon les obligeant à adopter une marche précautionneuse [18]. Quand il existe une atteinte rachidienne, le centre de gravité de ces patients est déplacé en avant et en bas dans un plan sagittal du fait de l'ankylose rachidienne, augmentant ainsi les risques de chutes. Une tentative de correction est essentiellement produite par les genoux et les chevilles plutôt que les hanches dans les positions statiques [19]. Elles peuvent être associées à des atteintes périphériques avec des enthèsites, des arthrites et des dactylites pouvant être responsables de destructions articulaires pouvant atteindre les membres inférieurs, perturbant alors les paramètres de marche $[5]$. 


\section{D’un point de vue cinématique :}

Les tests de marche réalisés chez 12 patients atteints de SA par Zebouni et al [18] à l'aide d'un électrogoniomètre retrouvaient une diminution significative des amplitudes articulaires de la hanche et du genou de manière bilatérale avec un pas plus court. Le rapport des angles hanche/genou ne différait pas du groupe témoin, ces anomalies expliquaient en partie la «démarche précautionneuse » observée chez ces patients. Ces anomalies n’étaient pas seulement dues aux douleurs et aux modifications articulaires mais également à une majoration de la transmission des vibrations dans le rachis due à l'ankylose [20]. Dans une étude comparative avec groupe témoin portant sur l'analyse de la marche par un système de capture de mouvements réalisée sur 12 patients atteints de SA, Del Din et al [5] montraient qu'il existait chez ces patients une augmentation de l'extension du rachis pendant le cycle de la marche. Ils retrouvaient également pendant la phase de charge une dorsiflexion de la cheville qui venait remplacer la flexion plantaire, ainsi qu'une diminution de la rotation du pelvis compensée par un pivotement du tronc autour de la charnière lombo-sacrée afin que ces patients puissent déplacer vers l'arrière leur centre de gravité, phénomène qui était majeur pendant la phase d'appui. Ils retrouvaient également une diminution des moments articulaires de rotation de la hanche et du genou pendant tout le cycle ainsi qu'une diminution de la flexion plantaire lors de la phase d'attaque du pied.

d. Troubles de la marche chez les patients atteints de Polyarthrite Rhumatoïde $(\mathrm{PR})$.

L'atteinte articulaire est polymorphe et globalement symétrique, débutant principalement par une atteinte des poignets et des articulations métacarpo-phalangiennes. Elle peut aussi bien atteindre les articulations des membres supérieurs que des membres inférieurs. On constate d'un point de vue radiographique l'apparition d'érosions périarticulaires au niveau des zones de réflexion de la synoviale puis de géodes intra osseuses juxta-articulaires, et secondairement l'apparition d'un pincement articulaire traduisant la destruction cartilagineuse et articulaire. 
Les déformations au niveau du pied comportent les hallux valgus, une exagération de la valgisation du talon, un effondrement de l'arc longitudinal médial et une diminution de hauteur de l'os naviculaire, elle sont les principales responsables des troubles de la marche et des chutes chez ces patients [21]. On constate une augmentation de la pression dans le 1er, le 2ème et le 5ème métatarsien (MT) secondaire à une marche adaptée aux douleurs articulaires et aux déformations des phalanges tarsiennes et de l'avant pied.

Les atteintes hétérogènes des articulations du genou et de la hanche sont également responsables d'anomalies sur le plan statique, ainsi que, dans les cas les plus sévères, l'atteinte du rachis cervical.

Les atteintes du genou comportent une déformation en flexion, le plus souvent en valgus, avec une rotation externe forcée. Elle peuvent-être accompagnées de kystes poplités volumineux, qui, lorsqu'ils se rompent, sont difficiles à différencier d'une phlébite [22].

L'atteinte des hanches engendre une coxite rhumatoïde, elle est présente chez environ $15 \%$ des patients limitant donc la flexion/extension lors du cycle de la marche. L'atteinte du rachis cervical, érosive, se manifeste au niveau de la charnière cervico-occipitale par une arthrite occipito-atloïdienne et altoïdo-axoïdienne (diastasis C1 et C2) avec un risque d'impression basilaire et une diminution des amplitudes articulaires engendrant une majoration de la kinésiophobie [23].

\section{D’un point de vue cinématique :}

Baan et al retrouvaient, dans une revue de la littérature reprenant 78 articles traitant des atteintes des membres inférieurs dans la PR, une diminution des amplitudes articulaires et de la puissance de la flexion/extension du genou et de la hanche, de l'abduction/adduction de cette dernière, de la flexion plantaire (notamment au décollement des orteils en début de phase oscillante) ainsi qu'une réduction de la dorsiflexion de la première métatarso-phalangienne [21]. Des mécanismes compensatoires ont été décrits tels qu'une rotation interne tibiale, un retard à l'élévation du talon, une éversion anormale de l'arrière pied, une augmentation de l'abduction de l'avant pied pendant la phase d'appui, une augmentation de la flexion de la hanche, et du genou dans un deuxième temps, lors de la phase oscillante, lorsque la perte de dorsiflexion de l'hallux devient trop importante [7,21]. 
Le moment d'une force par rapport à un point donné est une grandeur physique vectorielle traduisant l'aptitude de cette force à faire tourner un système mécanique autour de ce point, souvent appelé pivot.

Le moment articulaire correspond au produit de vecteur position décrit par Leardini et al [24], [figure 2] et de la force réactionnelle du sol lors de la marche.

Oda et al [9], dans une étude cinématique comparant 9 sujets atteints de PR avant et après la mise en place d'un anti TNF alpha, retrouvaient une augmentation du moment articulaire de l'extension de la hanche et de la dorsiflexion de la cheville durant le début de la phase d'appui, permettant une augmentation de la longueur du pas. En milieu de phase d'appui, le moment articulaire du genou diminuait afin de maintenir un équilibre optimal et, durant la fin de cette phase, le moment articulaire de la flexion de la hanche et de la cheville augmentait. Ces résultats avaient tendance à montrer que les anti-TNF alpha tendaient à normaliser la marche et que les destructions articulaires de la PR étaient également dues à une démarche pathologique entrainée par une sollicitation articulaire différente chez ces patients.

\section{e. L'accéléromètrie}

Le capteur d'accélération fixé par la ceinture en région lombaire (entre l'espace vertébral L3L4) enregistre tous les mouvements du centre de gravité au cours de la marche, une distance de 30 mètres est nécessaire afin d'enregistrer des phases de marche stabilisées et de s'affranchir des accélérations et des décélérations en début et fin de parcours. Les données sont ensuite analysées [Figure 3] automatiquement par un logiciel [14] :

Vitesse de la marche en $\mathrm{km} / \mathrm{h}$.

Cadence en cycle/s. Nombre de cycles de marche par seconde.

Longueur automatique en $\mathrm{m}$ : Obtenue en divisant la vitesse par la fréquence, elle correspond à la longueur du pas.

Régularité (sans unité) : Est la similarité du mouvement vertical mesurée sur des strides successives.

Symétrie (sans unité) : correspond à un index total de symétrie sur l'échantillon de marche analysé entre le pas gauche et le pas droit.

Puissance cranio-caudale en W/Kg : Mesure la kinésie du mouvement sur l'axe cranio-caudal. 
Puissance antéro-latérale en W/Kg : Mesure la kinésie du mouvement sur l'axe antéro-latéral. Puissance médio-latérale en W/Kg : Mesure la kinésie du mouvement sur l'axe médio-latéral. Onde de choc pathogène (sans unité) : L'onde de choc quantifie l'exposition du squelette aux sollicitations mécaniques de haute fréquence de types chocs et vibrations.

La symétrie et la régularité des strides: La détermination de ces deux variables fait appel au calcul de deux coefficients de corrélation $\mathrm{C} 1$ et $\mathrm{C} 2$. Ces deux coefficients sont obtenus en calculant la fonction d'autocorrélation sur le signal d'accélération verticale. C1 exprime la corrélation entre les accélérations correspondant aux mouvements des membres droits et gauches, C2 la corrélation entre l'accélération correspondant aux mouvements successifs de chaque membre. A partir de ces deux coefficients de corrélation, sont déduits les variables symétrie et régularité :

Symétrie : C1/C2 x 100(\%): Elle exprime la similitude des mouvements cranio-caudaux gauches et droits indépendamment des fluctuations des mouvements cranio-caudaux successifs de chaque membre.

Régularité : $(\mathrm{C} 1+\mathrm{C} 2)$ x 100 (score sur 200) : elle exprime la similitude des mouvements locomoteurs verticaux dans le temps.

Devant la distribution non gaussienne de ces paramètres, une transformation $Z$ de Fischer leur est appliquée pour leur attribuer une distribution normale.

Cet appareil a été utilisé dans de nombreuses études concernant des pathologies variées pouvant provoquer des troubles de la marche :

Mignardot et al [25], dans une étude prospective portant sur 259 patients âgés entre 66 et 75 ans n'ayant jamais chuté, retrouvaient un risque significatif de chute sur 2 composants principaux d'analyse (après une décomposition en valeurs d'une matrice de corrélation basée sur les variables enregistrées par l'accéléromètre) :

La kinésie globale de la marche (incluant notamment les vitesses, les puissances dans les trois axes, la fréquence, la longueur et la durée du pas) qui était prédictive d'une chute du patient dans les 6 premiers mois après l'inclusion. La régularité globale de la marche (incluant la symétrie, la régularité et le pourcentage d'ondes de chocs de hautes fréquences) qui était prédictive d'une chute dans les 6 à 12 mois après l'inclusion. 
Auvinet et al [26], dans une étude cas témoins observationnelle portant sur 52 patients atteints de fibromyalgie versus 52 patients appareillés dans chaque centre, utilisaient l'accéléromètrie afin d'identifier des variables qui différaient significativement de la population normale (puissance cranio-caudale, longueur de pas et fréquence du pas). Des corrélations entre ces variables et les différents scores d'évaluation des patients atteints de fibromyalgies étaient ensuite trouvées, ce qui avait permis d'isoler des sous-groupes de malades.

Paquet et al [27] montraient, en utilisant la même technique d'accéléromètrie et en comparant 22 sujets atteints de la maladie de parkinson (MP) avec des sujets sains, que les patients atteints de la MP réduisaient leurs vitesses de marche par un raccourcissement de la longueur du pas, associé à une diminution de la cadence. Ils constataient également une diminution de la régularité des pas et de l'activité crânio-caudale (reflet de l'état d'akinésie) ; ces deux dernières variables étaient fortement corrélées au score moteur mesuré par l'échelle d'évaluation unifiée (UPDRS).

f. Etude accéléromètrique des variations des troubles de la marche chez les patients atteints de PR et de SA après la mise en place d'un biomédicament.

Une évaluation des troubles de la marche avant et après la mise en place d'un biomédicament serait un bon critère d'évaluation de l'efficacité de ce dernier en ajoutant des critères objectifs à notre examen clinique. L'utilisation de l'accéléromètre est un outil simple, rapide (un test de marche dure en moyenne moins de 5 minutes) et non invasif de l'évaluation des paramètres de la marche.

A notre connaissance aucune étude ne compare l'évolution des données cinétiques de la marche avant et après la mise en place d'un biomédicament chez les patients atteints de SA. Il en est de même chez les patients atteints de PR, suivant le même protocole, aucune étude accéléromètrique n'a été réalisée ce jour.

\section{L'objectif de cette étude était double :}

L'objectif de cette étude était de montrer qu'il existait une amélioration des paramètres de la marche après la mise en place d'un biomédicament chez les patients atteints d'un rhumatisme inflammatoire.

L'objectif secondaire de cette étude était de montrer que ces modifications étaient corrélées à l'amélioration clinique des patients. 


\section{II) Patients et méthode}

a. Sélection

Il s'agissait d'une étude prospective observationnelle monocentrique réalisée d'aout 2014 à juin 2015 comportant deux branches :

Des patients atteints de SA axiale seule ou associée à des atteintes périphériques respectant les critères ASAS [Figures 4 et 5] et les recommandations pour la mise en place d'une biomédicament de la SFR 2014 [28].

Des patients atteints de PR respectant les critères de diagnostic ACR/EULAR 2010 [figure 6], nécessitant la mise en place d'un biomédicament ou un switch thérapeutique selon les recommandations de la SFR 2014 [29].

Ces patients étaient tous suivis en rhumatologie au CHU d'Amiens. Les critères d'exclusion comprenaient toutes les pathologies pouvant provoquer un trouble de la marche (notamment AVC, troubles de la vue, troubles de l'équilibre, neuropathie diabétique, atteinte arthrosique connue ou suspectée des membres inférieurs, antécédents d'intervention chirurgicale au niveau des membres inférieurs, canal lombaire étroit, infiltration intra articulaire dans le mois ou viscosuplémentations dans l'année, dérouillage matinal supérieur à $1 \mathrm{~h} 00$, antécédent de chute dans l'année précédente, prise de psychotrope et incapacité à la marche).

\section{b. Intervention}

Les patients devaient réaliser un test de marche dans un couloir sur un itinéraire précis mesurant 30 mètres, après leurs dérouillages matinaux. Les patients ne devaient ni parler, ni s'arrêter pendant ce test, auquel cas le test était renouvelé. Il était noté le détail du chaussage du patient (semelles incluses) et il était demandé au patient de revenir trois mois après pour un second test de marche avec le même chaussage et de réitérer le test à l'identique (couloir, distance et parcours).

Les données étaient ensuite analysées et enregistrées grâce au logiciel Locometrix par un évaluateur, un échantillon de marche stabilisée était alors sélectionné et analysé à J0 et trois mois plus tard. 


\section{c. Evaluation}

La moyenne de chaque paramètre de la marche, des scores d'activités (BASDAI, ASDAS, CRP et DAS 28) et des scores fonctionnels (HAQ et BASFI) était réalisée avant et après la mise en place du traitement. La significativité de la différence de leur moyenne était recherchée par un test non paramétrique de Wilcoxon.

Le critère de jugement principal était la démonstration d'une différence statistiquement significative de la puissance antéro-postérieure pour les SA et de la cadence pour la PR, critères considérés dans chaque pathologie comme les plus cliniquement significatifs et pertinents dans la littérature.

Une analyse « post hoc » était ensuite réalisée à la recherche d'une association entre les valeurs statistiquement significatives grâce au test de corrélation de Spearman appliqué sur les progressions en pourcentage des différents critères.

Une analyse en sous-groupes était également réalisée en classant les patients en répondeurs (amélioration de l'ASDAS CRP>1,1 et/ou du BASDAI de 50\% ou de plus de 2 points pour la SA et amélioration du DAS 28 de 1,2) et non répondeurs au traitement selon les recommandations de la SFR 2014 [28-29], afin de comparer leur évolution grâce à un test U de Mann-Whitney.

\section{d. Ethique}

Les patients ont été informés des données recherchées et de leurs buts par oral et par écrit, à cet égard, ils ont donné leur consentement libre et éclairé, conformément à la loi française. 


\section{III) Résultats}

a. Données observées à l'inclusion des patients

Vingt-trois patients ont été sélectionnés dans le groupe SA et 11 dans le groupe PR, les moyennes des paramètres obtenues grâce à un recueil de données à $\mathrm{J} 0$ sont présentées dans les tableaux 1 et 3 . Nous retrouvons les moyennes suivantes : une puissance antéro-postérieure de $1,40 \mathrm{~W} / \mathrm{kg}$ (avec un écart type (ET) à 0.59), une puissance médio-latérale à $1,12 \mathrm{~W} / \mathrm{kg}$ $(\mathrm{ET}=0.54)$ et une symétrie de 181,52 $(\mathrm{ET}=87.44)$ dans les SA et une cadence de 0,90 Cycle/s $(\mathrm{ET}=0.13)$ avec une vitesse de $3,7 \mathrm{~km} / \mathrm{h}(\mathrm{ET}=1.11)$ pour les $\mathrm{PR}$. Les normes des paramètres de marche (définies par une moyenne et un écart type) ne peuvent être comparées à ces résultats car ces derniers varient en fonction de l'âge, du sexe et du poids. Les résultats des paramètres de marche des patients recueillis grâce à l'accéléromètre, les scores d'activités (BASDAI, ASDAS CRP, DAS 28) ainsi que les scores fonctionnels (BASFI, HAQ) sont présentés dans les tableaux 2 et 4 .

\section{b. Données observées après traitement}

On retrouve chez les patients atteints de SA une évolution significative des puissances antéropostérieures $(0,70 \mathrm{~W} / \mathrm{kg})$ médio-latérales $(0,63 \mathrm{~W} / \mathrm{Kg})$ et de la symétrie (26). Chez les patients atteints de PR, seules des tendances sont retrouvées pour la cadence $(0,17$ cycles/s $\mathrm{p}=0,109)$ et la vitesse $(0,99 \mathrm{~km} / \mathrm{h} \mathrm{p}=0,083)$. Les matrices de corrélation de Spearman recherchant une corrélation entre les scores et les paramètres cliniques pour les résultats significatifs sont exposées dans les tableaux 5 et 6 . Aucune association statistiquement significative n'a été retrouvée. L'analyse en sous-groupes comparant l'évolution des paramètres de la marche chez les patients répondeurs et non répondeurs au traitement dans la SA est exposée dans le tableau 7. Nous retrouvons une différence significative à l'aide d'un test U de Mann Whitney pour la puissance antéro-postérieure $(1,86 \mathrm{~W} / \mathrm{Kg})$ et médio-latérale $(0,91 \mathrm{~W} / \mathrm{Kg})$. Etant donné l'absence de significativité pour les résultats concernant la PR et le faible effectif, il n'a pas été réalisé d'analyse en sous-groupes chez ces derniers. 


\section{IV) Discussion}

Notre étude s'intéressait à 34 patients atteints de RIC, 23 SA et 11 PR, le critère de jugement principal était validé pour la SA mais pas pour la PR.

\section{a. Concernant les SA}

On note tout d'abord une proportion d'hommes dans notre groupe SA s'élevant à seulement 48 $\%$. En effet, dans les années 1940, le sex-ratio homme/femme avait été estimé à 9 pour 1 . Les dernières études auraient tendance à se rapprocher d'un sex ratio homme/femme de 2/1 comme le montre l'étude GAZEL [30]. Ce chiffre est également dû au faible nombre de patients inclus. Il s'agissait à notre connaissance de la première étude comparant les résultats d'un test de marche avant et après la mise en place d'un biomédicament dans cette pathologie. Il existait certes des biais à cette étude notamment un biais de sélection, en effet, tous les patients étaient sélectionnés au CHU d'Amiens, il ne s'agissait pas obligatoirement de leur première biothérapie (il pouvait en effet s'agir d'un switch thérapeutique souvent moins efficace qu'une première biothérapie) et le nombre de sujets était faible, en cause notamment les critères d'exclusion sélectifs qui devaient exclure toutes autres pathologies pouvant intervenir dans un cycle de marche.

Il s'agissait également du plus grand effectif de patients atteints de SA bénéficiant d'une analyse cinétique de marche.

Notre étude montrait une amélioration significative des puissances antéro-postérieures, médiolatérales et totales après la mise en place d'un biomédicament. Cela tendrait à démontrer que le traitement de ces SA aurait tendance à normaliser certains paramètres de la marche, notamment par la libération du mouvement du rachis dans ces 2 plans et par une diminution de l'ankylose rachidienne.

L'augmentation de la puissance antéro-postérieure a plusieurs explications possibles :

- Une majoration des mouvements visant à corriger les anomalies du centre de gravité.

- Un pivotement du tronc autour du pelvis (donc dans le sens médio-latéral et antéropostérieur) et une diminution des douleurs qui augmenteraient la kinésie dans ces deux plans, elle-même étroitement liée aux puissances [19].

Metzinger et al [10] retrouvaient dans leurs études des diminutions statistiquement significatives des puissances crâniales caudales ainsi que de la fréquence, entre les sujets atteints de SA et les sujets sains. Malheureusement la significativité n'était pas atteinte pour les 
autres puissances compte tenu d'un effectif faible (8 patients). Ils attribuaient ces résultats à une kinésie plus faible (causée par la douleur chronique articulaire chez les patients atteints de SA).

Dans d'autres études, les anomalies des puissances dans les 3 axes ainsi que la symétrie, ressortaient comme facteurs potentiellement prédictifs de chutes à court terme [25]. Ceci pourrait donner un nouvel axe d'étude des risques de chutes chez les patients atteints de SA, étant donné la perturbation de ces paramètres chez ces malades.

DelDin.S et al [5] retrouvaient des différences importantes au niveau de la puissance craniocaudale entre sujets sains et sujets atteints de SA mais son étude qui se déroulait sur un faible effectif (9 patients SA) manque de puissance.

Notre étude manquait donc probablement de puissance pour retrouver ces anomalies.

L'amélioration de la symétrie pourrait quant à elle être due à l'asymétrie des atteintes articulaires des SA, à l'amélioration des douleurs et de la stabilité du centre d'équilibre de ces derniers après traitement par un biomédicament. [6]

La non-amélioration des ondes de choc pathogènes n'était pas surprenante puisque celle-ci était déjà décrite par Helliwell et Deldin et al $[5,20]$ et serait en partie responsable des anomalies constatées sur le plan sagittal par un déficit d'absorption des vibrations.

Aucune association statistique n'était retrouvée entre les scores d'évaluation de la SA et les puissances totales, antéro-posterieures, médio-latérales et la symétrie. Des études spécifiques semblent nécessaires pour démontrer ces associations.

L'analyse en sous-groupes retrouve une différence statistiquement significative entre les patients répondeurs et non-répondeurs pour les puissances médio-latérales et antéropostérieures, confirmant encore l'intérêt de ces paramètres pour le suivi des malades. 


\section{b. Concernant les PR}

Dans la littérature, une diminution de la vitesse, de la cadence, un pas plus court et un temps de double support plus long sont retrouvés chez les patients atteints de PR [21,31]. Rome et al ont montré que, hormis le temps de double support qui n'était pas calculé, ces critères avaient une excellente reproductibilité à plusieurs moments de la journée chez ces patients [32].

Carroll et al [33], dans une méta-analyse portant sur 19 études des paramètres spatiauxtemporels chez les patients atteints de PR, montraient que la modification de ces paramètres était principalement attribuée à une démarche qui vise à éviter les mouvements pouvant provoquer la douleur (démarche dite « antalgique ») avec une augmentation du temps de double support.

Plus récemment, dans une étude comparative portant sur 59 patients atteints de PR avec groupe témoin, il était retrouvé une diminution de la vitesse, de la cadence, de la longueur du pas et du temps de double support grâce à une analyse tridimensionnelle de la marche (3DGA) [8].

Une diminution significative de la puissance cranio-caudale, antéro-postérieure et de la puissance totale avait également été décrite en comparant l'analyse de la marche de 8 patients atteints de PR avec un groupe témoin à l'aide d'un accéléromètre [10].

Dans tous les cas, l'imputabilité de ces anomalies était toujours attribuée aux destructions articulaires et à la douleur.

Une tendance à l'amélioration de la vitesse de marche sous traitement était retrouvée dans notre étude comme cela avait également été retrouvé par Esbjörnsson et al [8]. En effet, cette équipe a comparé la modification des données cinétiques de 16 patients atteints de $\mathrm{PR}$ avant et après la mise en place d'une biothérapie et a mis en évidence une augmentation de la vitesse, de la cadence, du temps de double support, de la longueur du stride et du pas statistiquement significatives. En revanche, dans leur étude, la distance d'enregistrement de 10 mètres était trop courte pour s'affranchir de l'accélération et de la décélération lors de la marche.

L'augmentation de la cadence, quant à elle, n'atteignait pas un degré de significativité nécessaire pour être interprétable dans notre étude (contrairement à ce que retrouvent Metzinger et al [10]). On note que l'hypothèse que la cadence ne diffère pas d'un groupe témoin a été évoquée dans la littérature compte tenu de sa dépendance à l'effet pendulaire de la jambe qui est directement relié à la stature du patient [4], mais cela ne tient pas compte d'une atteinte articulaire de la hanche. 
Cette amélioration de la cadence et de la vitesse, pourrait s'expliquer par une diminution des douleurs et du risque de chutes, d'autres études sont nécessaires pour le confirmer.

Les différentes puissances montraient une tendance à l'amélioration après traitement mais l'inclusion d'un nombre plus important de patients semblait nécessaire pour retrouver une significativité. Aucune corrélation n'est retrouvée entre la vitesse et le DAS 28.

\section{V) Conclusion}

Pour les patients atteints de SA, les puissances antéro-postérieures, médio-latérales ainsi que la symétrie, semblent être des données intéressantes à suivre dans l'évolution de la maladie. Notre étude prouvait que ces dernières augmentaient de manière significative chez les malades traités. Ces deux puissances évoluent de manière différente entre les patients répondeurs et non répondeurs.

Concernant les patients atteints de PR, seules des tendances à l'amélioration étaient observées pour l'évolution de la cadence et de la vitesse après la mise en place d'un biomédicament, aucune association statistique n'était retrouvée pour ces critères avec le DAS 28, une étude avec un effectif plus important pourrait confirmer ce résultat. Ce dernier paramètre pourrait peut-être représenter un marqueur pertinent pour évaluer l'efficacité d'un traitement au cours de la PR. 
VI) $\underline{\text { Annexes }}$

Figure 1 : Le cycle de la marche

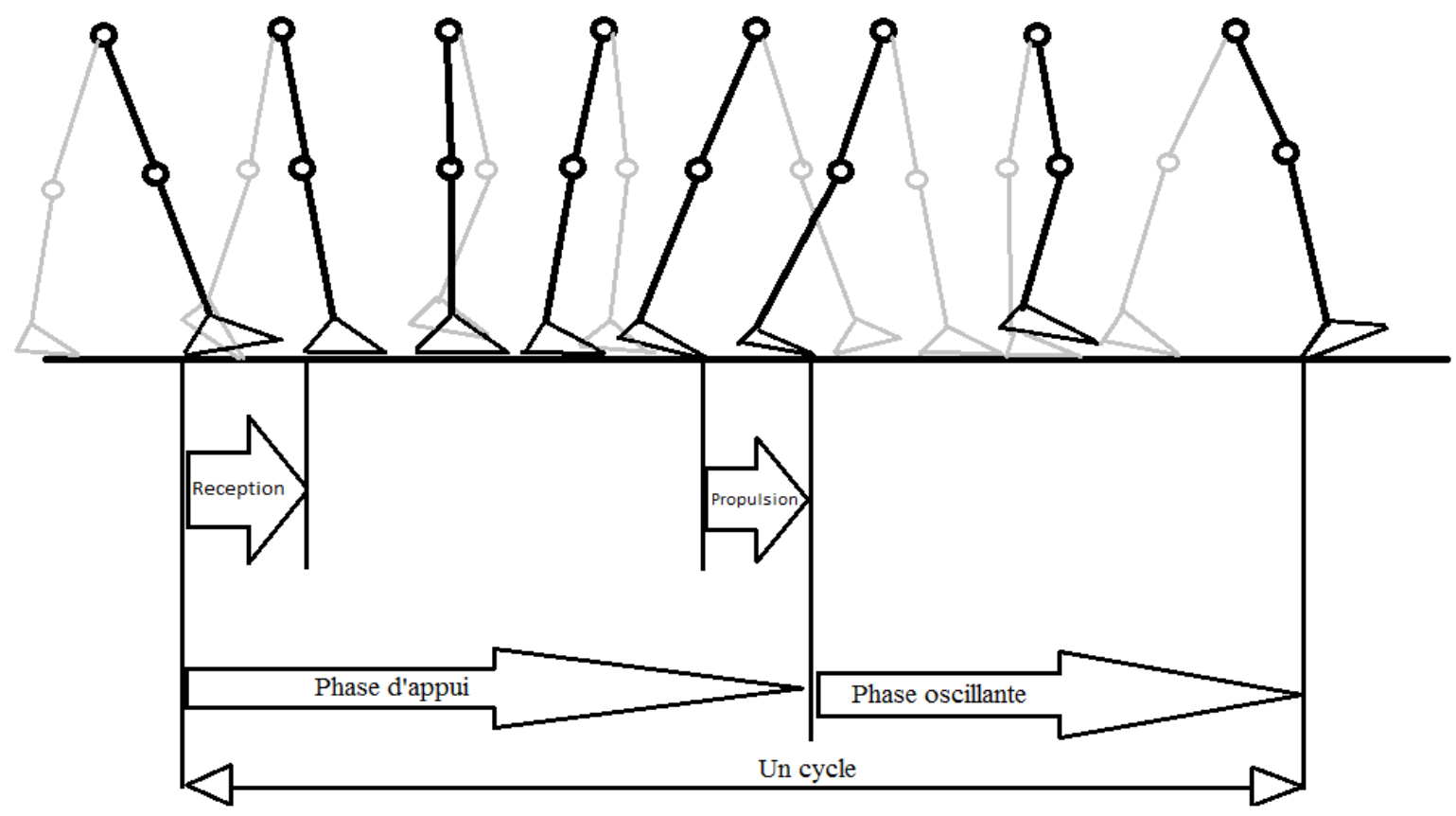

Figure 2: Vecteurs positions standardisés selon Leardini

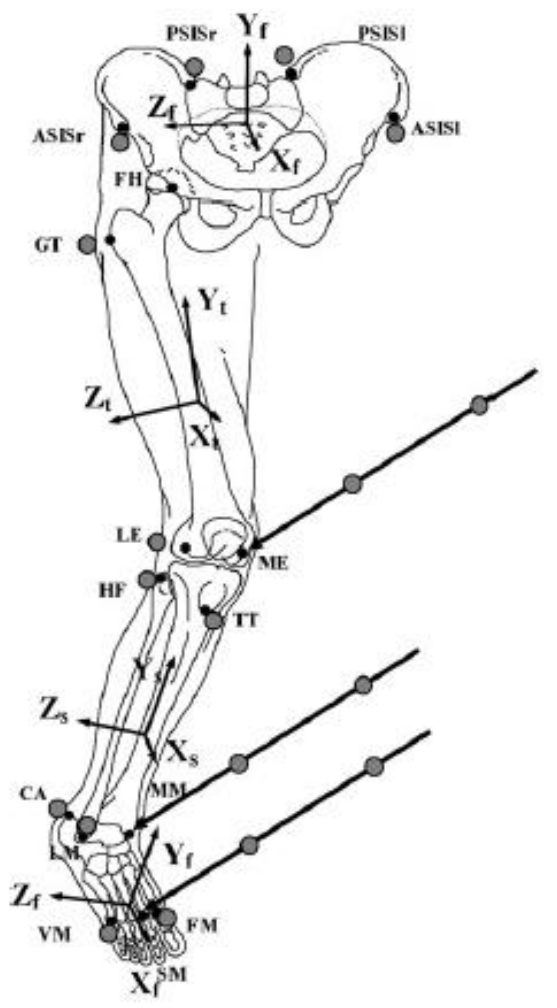


Figure 3: Interface de l'accéléromètre

\section{Mesures de la marche \\ Man 20-49y \\ Paramètres cinématiques des foulées: Patient Moyenne

\begin{tabular}{llll} 
Vitesse & $(\mathrm{km} / \mathrm{h})$ & 2.43 & 5.65 \\
\hline Longueur automatique : $(\mathrm{m})$ & & 0.86 & 1.61 \\
\hline
\end{tabular}

\begin{tabular}{|c|c|c|}
\hline Cadence des cycles (cycles/s): & 0.78 & 0.95 \\
\hline \multicolumn{3}{|l|}{ Paramètres de coordination: } \\
\hline Régularité (plus de 300): & 80 & 338 \\
\hline Symétrie (plus de 200): & 0 & 243 \\
\hline \multicolumn{3}{|l|}{ Paramètres des contraintes } \\
\hline Onde de choc pathogène (moins de $11 \%$ ): & 33.10 & 10.64 \\
\hline \multicolumn{3}{|l|}{ Paramètres énergétiques: } \\
\hline Puissance crenio-caudale $(\mathrm{W} / \mathrm{kg})$ : & 1.95 & 5.46 \\
\hline Puissance antéro-postérieure (W/kg) & 0.81 & 2.04 \\
\hline Puissance mádio-latérale $(\mathrm{W} / \mathrm{kg})$ : & 0.75 & 1.21 \\
\hline Puissance totale (W/kg): & 3.51 & 8.71 \\
\hline Consommation d'oxygène (ml/min/kg): & 13 & 17 \\
\hline Consommation d'énergie (kJ/h/kg): & 12.64 & 31.36 \\
\hline
\end{tabular}

\section{Synthèse des résultats}

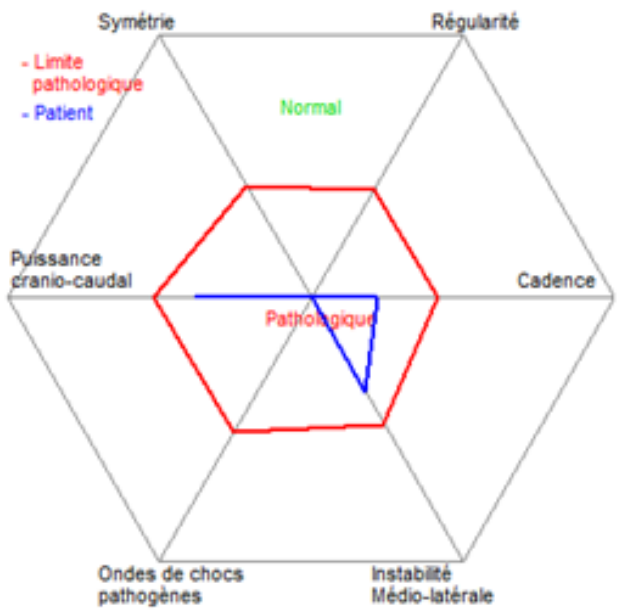


Figure 4 : Critères ASAS des spondylarthrites ankylosantes axiales

\begin{tabular}{|l|l|}
\hline Age inférieur à 45 ans et : & $\underline{\text { Sacro-iliite à l'imagerie : }}$ \\
Sacro-iliite à l'imagerie avec au moins 1 signe & $\begin{array}{l}\text { Inflammation fortement évocatrice de sacro- } \\
\text { iliite à l'IRM } \\
\text { Ou }\end{array}$ \\
HLA-B27 positif + au moins 2 signes de SA & $\begin{array}{l}\text { Une sacro-iliite radiographique définie suivant } \\
\text { les critères de New-York modifiés }\end{array}$ \\
& $\begin{array}{l}\text { Signes de spondylarthrite : } \\
\text { Rachialgie inflammatoire } \\
\text { Arthrite } \\
\text { Enthésite (talon) } \\
\text { Uvéite } \\
\text { Dactylite } \\
\text { Psoriasis } \\
\text { Maladie de Crohn/Rectocolite hémorragique } \\
\text { Bonne réponse aux AINS } \\
\text { Antécédent familial de SA } \\
\text { HLA-B27 positif } \\
\text { CRP augmentée }\end{array}$ \\
\hline
\end{tabular}

Figure 5 : critères ASAS des spondylarthrites ankylosantes périphériques.

\begin{tabular}{|l|lc|}
\hline Age inférieur à 45 ans et : & (A) : & (B) : \\
Arthrite(s) & Uvéite & Arthrite(s) \\
Ou enthésite(s) & Psoriasis & Enthesite(s) \\
Ou dactylite(s) & MICI & Dactylite(s) \\
& Infection & Rachialgies inflammatoires \\
Avec au moins un critère de SPA (A) & HLA-B27 & ATCD familiale de SA \\
ou au moins deux critères de SPA (B) & Sacro-iliite & \\
& & \\
& & \\
\hline
\end{tabular}


Figure 6: Critère diagnostic ACR/EULAR 2010:

En l'absence d'érosions typiques radiologiques, le diagnostic est retenu pour un score au moins égal à 6

\begin{tabular}{|l|l|}
\hline Type d'atteinte articulaire (0-5) & \\
\hline 1 articulation moyenne ou grosse & 0 \\
\hline 2-10 articulations moyennes ou grosses & 1 \\
\hline 1-3 petites articulations & 2 \\
\hline $4-10$ petites articulations & 3 \\
\hline$>10$ articulations (au moins 1 petite articulation) & 5 \\
\hline Sérologie (0-3) & \\
\hline Ni FR ni ACPA & 0 \\
\hline Au moins un test faiblement positif & 2 \\
\hline Au moins un test forcement positif & 3 \\
\hline Durée de la synovite (0-1) & \\
\hline$<6$ semaines & 0 \\
\hline$>6$ semaines & 1 \\
\hline Marqueurs de l'inflammation (0-1) & \\
\hline Ni CRP ni VS élevée & 1 \\
\hline CRP ou VS élevée & \\
\hline
\end{tabular}


$\underline{\text { Tableau } 1 \text { : Paramètres cliniques et biologiques des patients atteints de SA à J0 }}$

\begin{tabular}{|l|l|}
\hline Données & Moyenne à J0 SA \\
\hline$n$ & 23 \\
\hline IMC & $28.95{\mathrm{~kg}-\mathrm{m}^{\wedge} 2}^{\circ}$ \\
\hline AGE & 43.39 \\
\hline SEXE masculin & $48 \%$ \\
\hline BASFI & 5.2 \\
\hline ASDAS CRP & 3.6 \\
\hline BASDAI & 5.0 \\
\hline AINS à J0 & $58 \%$ \\
\hline Tabagisme actif & $45 \%$ \\
\hline HLAB27+ & $63 \%$ \\
\hline Initiation & $91 \%$ \\
\hline
\end{tabular}


$\underline{\text { Tableau 2: Paramètres de marche et scores d'activités des patients atteints de SA à J0 et J90 }}$

\begin{tabular}{|c|c|c|c|c|}
\hline $\begin{array}{l}\text { Paramètre de } \\
\text { marche }\end{array}$ & J0 (ET) & J90 (ET) & Différence & p-value \\
\hline Vitesse en $\mathrm{km} / \mathrm{h}$ & $4.20(0,53)$ & $4.30(0,77)$ & 0.10 & 0.429 \\
\hline Cadence en cycle/s & $0.92(0,063)$ & $0.93(0,092)$ & 0.01 & 0.699 \\
\hline $\begin{array}{l}\text { Longueur } \\
\text { automatique en m }\end{array}$ & $1.26(0,11)$ & $1.28(0,14)$ & 0.02 & 0.280 \\
\hline Régularité & $247.91(51,79)$ & $253.13(55,63)$ & 5.22 & 0.484 \\
\hline Symétrie & $181.52(87,44)$ & $207.52(60,94)$ & 26 & 0.020 \\
\hline $\begin{array}{l}\text { Puissance totale en } \\
\mathrm{W} / \mathrm{kg}\end{array}$ & $5.30(2,37)$ & $7.05(5,54)$ & 1.75 & 0.029 \\
\hline $\begin{array}{l}\text { Puissance cranio- } \\
\text { caudale en } \mathrm{W} / \mathrm{kg}\end{array}$ & $2.78(1,55)$ & $3.20(2,23)$ & 0.42 & 0.308 \\
\hline $\begin{array}{l}\text { Puissance antéro- } \\
\text { postérieure en W/kg }\end{array}$ & $1.40(0,59)$ & $2.10(1,99)$ & 0.70 & 0.006 \\
\hline $\begin{array}{l}\text { Puissance médio- } \\
\text { latérale en } \mathrm{W} / \mathrm{kg}\end{array}$ & $1.12(0,54)$ & $1.75(1,66)$ & 0.63 & 0.003 \\
\hline $\begin{array}{l}\text { Onde de choc } \\
\text { pathogène en } \%\end{array}$ & $16.01(5,28)$ & $15.81(5,88)$ & -0.20 & 0.867 \\
\hline BASDAI & $5.0(1,33)$ & $2.6(0,67)$ & -2.4 & $<0.0001$ \\
\hline BASFI & $5.2(1,65)$ & $4.4(2,28)$ & -0.8 & 0.095 \\
\hline ASDAS CRP & $3.6(2,28)$ & $3.4(2,33)$ & -0.2 & 0.444 \\
\hline
\end{tabular}

$($ ET) $=$ Ecart Type 
$\underline{\text { Tableau } 3 \text { : Paramètres cliniques et biologiques de patients atteints de PR à J0 }}$

\begin{tabular}{|l|l|}
\hline Données & Moyenne à J0 \\
\hline$n$ & 11 \\
\hline IMC & 21.58 \\
\hline AGE & 56 \\
\hline SEXE masculin & $27 \%$ \\
\hline DAS 28 CRP & 3.95 \\
\hline HAQ & 1.30 \\
\hline Méthotrexate à J0 & $73 \%$ \\
\hline Tabagisme actif & $36 \%$ \\
\hline FR + & $64 \%$ \\
\hline CCP + & $81 \%$ \\
\hline Initiation & $73 \%$ \\
\hline
\end{tabular}


$\underline{\text { Tableau } 4 \text { : Paramètres de marche des patients atteints de PR à J0 et J90 }}$

\begin{tabular}{|c|c|c|c|c|}
\hline $\begin{array}{l}\text { Paramètre de } \\
\text { marche }\end{array}$ & J0 (ET) & J90 (ET) & $\begin{array}{l}\text { Différence } \\
\text { J90-J0 }\end{array}$ & p-value \\
\hline Vitesse en $\mathrm{km} / \mathrm{h}$ & $3.7(1,11)$ & $4.69(1,04)$ & 0.99 & 0.083 \\
\hline $\begin{array}{l}\text { Cadence en } \\
\text { cycle/s }\end{array}$ & $0.90(0,13)$ & $1.07(0,30)$ & 0.17 & 0.109 \\
\hline $\begin{array}{l}\text { Longueur } \\
\text { automatique en } \\
\mathrm{m}\end{array}$ & $1.15(0,21)$ & $1.26(0,14)$ & 0.11 & 0.306 \\
\hline Régularité & $196,36(83,06)$ & $240,36(71,36)$ & 44 & 0.266 \\
\hline Symétrie & $173,22(53,29)$ & $171,22(74,05)$ & -2 & 0.965 \\
\hline $\begin{array}{l}\text { Puissance totale } \\
\text { en } \mathrm{W} / \mathrm{kg}\end{array}$ & $5.47(3,33)$ & $6.39(3,28)$ & 0.92 & 0.351 \\
\hline $\begin{array}{l}\text { Puissance cranio- } \\
\text { caudale en } \mathrm{W} / \mathrm{kg}\end{array}$ & $2.76(2,07)$ & $3.28(1,64)$ & 0.52 & 0.351 \\
\hline $\begin{array}{l}\text { Puissance antéro- } \\
\text { postérieure en } \\
\mathrm{W} / \mathrm{kg}\end{array}$ & $1.47(0,76)$ & $1.88(0,97)$ & 0.41 & 0.307 \\
\hline $\begin{array}{l}\text { Puissance médio- } \\
\text { latérale en w/kg }\end{array}$ & $1.24(0,71)$ & $1.23(0,83)$ & -0.01 & 0.894 \\
\hline $\begin{array}{l}\text { Onde de choc } \\
\text { pathogène en } \%\end{array}$ & $20.8(6,29)$ & $17.7(9,40)$ & -3.1 & 0.350 \\
\hline HAQ & $1.30(0,99)$ & $1.13(0,97)$ & -0.17 & 0.722 \\
\hline DAS 28 & $3.95(1,05)$ & $2.98(1,30)$ & -0.97 & 0.048 \\
\hline
\end{tabular}

$(\mathrm{ET})=$ Ecart Type 
$\underline{\text { Tableau 5: Matrice de corrélation de Spearman pour la SA }}$

\begin{tabular}{|l|l|l|l|l|}
\hline Score & $\begin{array}{l}\text { Puissance totale } \\
(\mathrm{p})\end{array}$ & $\begin{array}{l}\text { Puissance antéro- } \\
\text { postérieure }(\mathrm{p})\end{array}$ & $\begin{array}{l}\text { Puissance médio- } \\
\text { latérale }(\mathrm{p})\end{array}$ & $\begin{array}{l}\text { Symétrie } \\
(\mathrm{p})\end{array}$ \\
\hline BASDAI & $0.082(0,71)$ & $0.176(0,42)$ & $-0.353(0,10)$ & $0.286(0,19)$ \\
\hline BASFI & $0.205(0,346)$ & $0.168(0,44)$ & $-0.144(0,510)$ & $0.344(0,108)$ \\
\hline
\end{tabular}

$(p)=p$-value

$\underline{\text { Tableau } 6 \text { : Matrice de corrélation de Spearman pour la PR }}$

\begin{tabular}{|l|l|}
\hline Score & DAS 28 $(\mathrm{p})$ \\
\hline Vitesse & $-0.245(0,468)$ \\
\hline Cadence & $-0,173(0,614)$ \\
\hline
\end{tabular}

$(\mathrm{p})=\mathrm{p}$-value

$\underline{\text { Tableau } 7 \text { : Analyse en sous-groupes de l'évolution des paramètres de la marche en fonction }}$ des scores d'activités

\begin{tabular}{|c|c|c|c|c|}
\hline & $n=$ & $\begin{array}{l}\Delta=\text { Puissance antéro- } \\
\text { postérieure }(\mathrm{W} / \mathrm{kg})\end{array}$ & $\begin{array}{l}\Delta=\text { Puissance médio- } \\
\text { latérale }(\mathrm{W} / \mathrm{kg})\end{array}$ & $\Delta=$ Symétrie \\
\hline (1) $\triangle$ ASDAS CRP $<1,1$ & 16 & $\mathbf{0 , 1 3}$ & $\mathbf{0 , 2 9}$ & 8,3 \\
\hline (2) $\triangle$ ASDAS CRP $>1,1$ & 7 & 1,99 & 1,20 & 66,3 \\
\hline $\mathrm{p}^{*}$ & & 0,003 & 0,006 & 0,140 \\
\hline $\begin{array}{l}\text { (3) } \Delta \text { BASDAI }<2 \text { ou } \\
<50 \%\end{array}$ & 8 & 0,52 & 0,36 & 46,25 \\
\hline 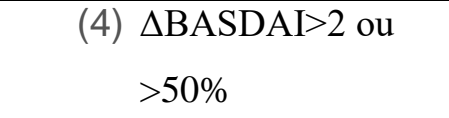 & 15 & 0,73 & 0,66 & 13,4 \\
\hline $\mathrm{p}^{* *}$ & & 0,730 & 0,605 & 0,862 \\
\hline
\end{tabular}

$\Delta=$ différence des moyennes entre $\mathrm{J} 90$ et $\mathrm{J} 0$

$\mathrm{p}=\mathrm{p}$-value de la différence entre les sous-groupes 1 et $2\left(\mathrm{p}^{*}\right)$ et 3 et $4\left(\mathrm{p}^{* *}\right)$ après réalisation d'un test

U de Man Whitney 


\section{VII) Bibliographie}

[1] Marey EJ. De la locomotion terrestre chez les bipèdes et les quadrupèdes. Paris : Martinet imprimeur-librairie ; 1873.p.42-80.

[2] Muybridge E. Muybridge's complete human and animal locomotion. New York : Dover Publication; 1887.vol.1,2,3.

[3] Fransen M, Heussler J, Margiotta E, Edmonds J. Quantitative gait analysis comparison of rheumatoid arthritic and non-arthritic subjects. Aust J Physiother. 1994;40:191-9.

[4] Eppeland SG, Myklebust G, Hodt-Billington C, Moe-Nilssen R. Gait patterns in subjects with rheumatoid arthritis cannot be explained by reduced speed alone. Gait Posture. 2009;29: 499-503.

[5] Del Din S, Carraro E, Sawacha Z, Guiotto A, Bonaldo L, Masiero S et al. Impaired gait in ankylosing spondylitis. Med Biol Eng Comput. 2011;49:801-9.

[6] Mangone M, Scettri P, Paoloni M, Procaccianti R, Spadaro A, Santilli V. Pelvis-shoulder coordination during level walking in patients with ankylosing spondylitis. Gait Posture. 2011;34:1-5.

[7] Broström EW, Esbjörnsson AC, von Heideken J, Iversen MD. Gait deviations in individuals with inflammatory joint diseases and osteoarthritis and the usage of threedimensional gait analysis. Best Pract Res Clin Rheumatol. 2012;26:409-22.

[8] Esbjörnsson AC, Rozumalski A, Iversen MD, Schwartz MH, Wretenberg P, Broström EW. Change in Gait Deviation Index after anti-tumour necrosis factor- $\alpha$ treatment in individuals with rheumatoid arthritis : a pilot study. Scand J Rheumatol. 2014;43:124-131.

[9] Oda R, Fujiwara H, Tokunaga D, Nakamura S, Taniguchi D, Kawahito Y et al. How do anti-TNF therapies affect gait function in patients with rheumatoid arthritis ? Int J Rheum Dis. 2014; $17: 57-62$.

[10] Metzinger R, Vaillant J, Gaudin P, Vuillerme N, Sugny S, Franco C et al. Rhumatismes inflammatoires : performances d'équilibre et de locomotion. Kinesitherapie, la revue. 2014;13:26-34. 
[11] Murray HC, Elliott C, Barton SE, Murray A. Do patient with ankylosing spondylitis have poorer balance than normal subjects. Rheumatology (Oxford). 2000;39:497-500.

[12] Bot SDM, Caspers M, Van Royen BJ, Toussaint HM, Kingma I. Biomechanical analysis of posture in patients with spinal kyphosis due to ankylosing spondylitis : a pilot study. Rheumatology (Oxford). 1999;38:441-3.

[13] Vergara ME, O’Shea FD, Inman RD, Gage WH. Posturale control is altered in patients with ankylosing spondylitis. Clin Biomech. 2012;27:334-40.

[14] Auvinet B, Chaleil D, Barrey E. Accelerometric gait analysis for use in hospital outpatients. Rev Rhum Engl Ed 1999;66:389-97.

[15] Auvinet B, Berrut G, Touzard C, Moutel L, Collet N, Chaleil D et al. Reference data for normal subjects obtained with an accelerometric device. Gait and Posture. 2002;16:124-34.

[16] Reddy NP, Rothschild BM, Verrall E, Joshi A. Noninvasive measurement of acceleration at the knee joint in patients with rheumatoid arthritis and spondyloarthropathy of the knee. Ann Biomed Eng. 2001;29:1106-11.

[17] Zochling J, Van Der Heijde D, Burgos-Vargas R, Collantes E, Davis JC, Dijkmans B Jr et al. ASAS/EULAR recommendations for the management of ankylosing spondylitis. Ann Rheum Dis. 2006;65:442-52.

[18] Zebouni L, Helliwell PS, Howe A, Wright V. Gait analysis in ankylosing spondylitis. Ann Rheum Dis. 1992;51:898-9.

[19] Guiotto A, Del din S, Carraro E, Sawacha Z, Leopaldi G, Baldi L et al. Kinematics and kinetics analysis of gait in ankylosing spondylitis subjects. Gait posture. 2009;30:42.

[20] Helliwell PS, Smeathers JE, Wright V. Shock absorption by the spinal column is normal and in ankylosing spondylitis. Proc Inst Mech Eng H. 1989;203:187-90.

[21] Baan H, Dubbeldam R, Anand V, Nene A, Van de laar M. Gait analysis of the lower limb in patients with rhumatoid arthrtitis : A systematic review. Arthritis Rheum. 2012;41:768-88.

[22] Abemethy PJ. Surgery of the rheumatoid knee. Ann Rheum Dis. 1990;49:830-6. 
[23] Pillon F Michiels Y. Manifestation cliniques de la polyarthrite rhumatoïde. Actualités pharmaceutiques. 2013;52:3-5.

[24] Leardini A, Sawacha Z, Paolini G, Ingrosso S, Nativo R, Benedetti MG et al. A new anatomically based protocol for gait analysis in children. Gait Posture. 2007;26:560-71.

[25] Mignardot JB, Deschamps T, Barrey E, Auvinet B, Berrut G, Cornu C et al. Gait disturbances as specific predictive markers of the first fall onset in elderly people : a two-year prospective observational study. Front Aging Neurosci. 2014;6:22.

[26] Auvinet B, Chaleil D, Cabane J, Dumolard A, Hatron P, Juvin R et al. The interest of gait markers in the identification of subgroups among fibromyalgia patients. BMC Musculoskelet Disord. 2011;12:258.

[27] Paquet JM, Auvinet B, Chaleil D, Barrey E. Analysis of gait disorders in Parkinson's disease assessed with an accelerometer. Rev Neurol (Paris). 2003;159:786-9.

[28] Wendling D, Lukas C, Paccou J, Claudepierre P, Carton L, Combe B et al. Recommandations de la Société francaise de rhumatologie (SFR) pour la prise en charge en pratique courante des malades atteints de spondyloarthrite. Rev rhum. 2014;81:6-15.

[29] Gaujoux-Viala C, Gossec L, Cantagrel A, Dougados M, Fautrel B, Mariette X et al. Recommandations de la Société française de rhumatologie pour la prise en charge de la polyarthrite rhumatoïde. Rev Rhum. 2014;81:303-12.

[30] Costantino F, Talpin A, Said-Nahal R, Goldberg M, Henny J, Chiocchia G et al. Prevalence of spondyloarthritis in reference to HLA-B27 in the French population : results of the GAZEL cohort. Ann Rheum Dis. 2015;74:689-93.

[31] Rome K, Dixon J, Gray M, Woodley R. Evaluation of static and dynamic postural stability in established rheumatoid arthritis : exploratory study. Clin Biomech. 2009;24:524-6.

[32] Rome K, Hanchard NC. Within-day reliability of temporal-spatial gait parameters associated with rheumatoid arthritic feet. Musculoskeletal Care. 2005;3:17-23.

[33] Carroll M, Parmar P, Dalbeth N, Boocock M, Rome K. Gait characteristics associated with the foot and the ankle in inflammatory arthritis : a systematic review and meta-analysis. BMC Musculoskelet Disord. 2015;16:134. 




\section{Summary}

Evolution of the walk parameters after biologic treatment during chronic inflammatory rheumatism (CIR).

Objectives: The aim of this study is to observe modification of the gait parameters of patients with CIR (Rheumatoid arthritis (RA) or Ankylosing spondylitis (AS)) after initiation of treatment with a biologic.

Study design: This is a single-center, prospective, observational study.

Patients: Patients with AS or RA were included upon initiation of the biologic.

Methods: A walking test was carried at D0 and 3 months. The primary endpoint was the demonstration of a statistically significant difference in anterior-posterior power for patients with AS and in cadence for patients with RA.

Results: Thirty-four patients were included. The study found a increase of anterior-posterior power $(0.7 \mathrm{~W} / \mathrm{kg} ; p=0.006)$ medio-lateral power $(0.7 \mathrm{~W} / \mathrm{kg} ; p=0.003)$ and symmetry $(+26$ $p=0.02$ ) in patients with AS, a significantly different evolution of the two powers depending on the response to treatment has been prouved $(0,13 \mathrm{~W} / \mathrm{Kg}$ and $0,29 \mathrm{~W} / \mathrm{Kg}$ for differences of anterior-posterior and medio-lateral powers betwen D0 and 3 months for nonresponders patients ; $1,99 \mathrm{~W} / \mathrm{Kg}$ et $1,32 \mathrm{~W} / \mathrm{Kg}$ for responders patients) and a trend towards increased cadence $(0.17$ cycles $/ \mathrm{s} ; p=0.109)$ and speed $(0,99 \mathrm{~km} / \mathrm{h} \mathrm{p}=0,083)$ in patients with RA.

\section{Conclusions:}

In patients with AS: The improvement in anterior-posterior and medio-lateral power can be explained by an increase in movements intended to correct abnormalities in the center of gravity. It seems to be interesting data points to follow up over the course of the disease. For RA patients; this study found a trend of improvement in cadence. These results could be explained by a decreased pain. A study with a larger sample size could confirm this results.

\section{Keywords:}

Gait disorder, accelerometer, inflammatory rheumatism, ankylosing spondylitis, rheumatoid arthritis, biologic. 
Evolution des paramètres de la marche sous traitement par biomédicament au cours des rhumatismes inflammatoires chroniques (RIC).

Introduction : l'objectif de cette étude était d'observer l'évolution des paramètres de marche chez les patients atteints de RIC sous biomédicament.

Matériel et méthode : Étude observationnelle prospective monocentrique. Inclusion de patients atteints de spondylarthrite ankylosante (SA) et de polyarthrite rhumatoïde (PR). Un test de marche était réalisé à l'introduction du biomédicament et à J90. Le critère de jugement principal était une différence significative de la puissance antéro-postérieure pour les SA et de la cadence pour les PR.

Résultats : Trente-quatre patients ont été inclus. On retrouvait une augmentation de la puissance antéro-postérieure $(0,7 \mathrm{~W} / \mathrm{Kg})$, médio-latérale $(0,6 \mathrm{~W} / \mathrm{Kg})$ et de la symétrie (26) ainsi qu'une évolution significativement différente des deux puissances en fonction de la réponse au traitement dans la SA $(0,13 \mathrm{~W} / \mathrm{Kg}$ et $0,29 \mathrm{~W} / \mathrm{Kg}$ pour les différences des puissances antéroposterieures et médio-latérales entre J0 et J90 dans le groupe non répondeurs; $1,99 \mathrm{~W} / \mathrm{Kg}$ et $1,32 \mathrm{~W} / \mathrm{Kg}$ pour le groupe répondeurs) et une tendance à l'augmentation de la cadence $(0,17$ cycles/s ; $\mathrm{p}=0,109)$ et de la vitesse $(0,99 \mathrm{~km} / \mathrm{h} \mathrm{p}=0,083)$ dans la $P R$.

Discussion : Dans les SA, l'amélioration des puissances antéro-postérieures et médio-latérales pouvait s'expliquer par une majoration des mouvements visant à corriger les anomalies du centre de gravité. Dans les PR, l'amélioration de la cadence et de la vitesse pourrait s'expliquer par une diminution des douleurs.

\section{$\underline{\text { Conclusion : }}$}

Pour les SA, la puissance antéro-postérieure semble être une donnée intéressante à suivre dans l'évolution de la maladie ; pour les PR un effectif plus important est nécessaire pour conclure.

\section{Mots clés :}

Troubles de la marche, accéléromètre, rhumatisme inflammatoire, spondylarthrite ankylosante, polyarthrite rhumatoïde, biomédicament. 